Virginia Commonwealth University VCU Scholars Compass

2012

\title{
Magnetism of electrons in atoms and superatoms
}

Victor Medel

Virginia Commonwealth University

J. Ulises Reveles

Virginia Commonwealth University, jureveles@vcu.edu

Shiv N. Khanna

Virginia Commonwealth University, snkhanna@vcu.edu

Follow this and additional works at: http://scholarscompass.vcu.edu/phys_pubs

Part of the Physics Commons

Medel, V., Reveles, J. U., \& Khanna, S. N. Magnetism of electrons in atoms and superatoms. Journal of Applied Physics, 112, 064313 (2012). Copyright (C) 2012 American Institute of Physics.

\section{Downloaded from}

http://scholarscompass.vcu.edu/phys_pubs/126

This Article is brought to you for free and open access by the Dept. of Physics at VCU Scholars Compass. It has been accepted for inclusion in Physics Publications by an authorized administrator of VCU Scholars Compass. For more information, please contact libcompass@vcu.edu. 


\title{
Magnetism of electrons in atoms and superatoms
}

\author{
Victor Medel, J. Ulises Reveles, and Shiv N. Khanna ${ }^{\text {a) }}$ \\ Department of Physics, Virginia Commonwealth University, Richmond, Virginia 23284, USA
}

(Received 25 June 2012; accepted 15 August 2012; published online 20 September 2012)

\begin{abstract}
The quantum states of electrons in small symmetric metallic clusters are grouped into shells similar to the electronic shells in free atoms, leading to the conceptual basis for defining superatoms. The filling of the electronic shells in clusters, however, do not follow Hund's rule and usually result in non-magnetic species. It is shown that by embedding a transition metal in group II atoms, one can stabilize superatoms with unpaired electronic supershells. We demonstrate this intriguing effect through electronic structure studies of $\operatorname{MnSr}_{n}(n=6-12)$ clusters within first principles generalized gradient calculations. The studies identify an unusually stable magnetic $\mathrm{MnSr}_{9}$ species with a large exchange splitting of $1.82 \mathrm{eV}$ of the superatomic D-states. It is shown that the exchange split d-states in the Mn atom induce exchange splitting in S and D superatomic shells because of the hybridization between orbitals of selected parity. The magnetic $\mathrm{MnSr}_{9}$ cluster with 25 valence electrons has filled $1 \mathrm{~S}^{2}, 1 \mathrm{P}^{6}, 1 \mathrm{D}^{10}, 2 \mathrm{~S}^{2}$ shells, making it highly stable, and an open shell of 5 unpaired D electrons breeding the magnetic moment. The stable cluster is resistant to collapse as two motifs are united to form a supermolecule. (C) 2012 American Institute of Physics.
\end{abstract}

[http://dx.doi.org/10.1063/1.4752471]

\section{INTRODUCTION}

The electronic states in atoms are grouped into shells characterized by $n l$ quantum numbers where the orbital angular quantum number $l$ can assume values $0,1, \ldots, n-1$ leading to the shell sequence $1 s, 2 s, 2 p, 3 s, 3 p, 3 d, 4 s, \ldots$ Atoms with filled shells (Octet rule) exhibit enhanced stability as manifested through their electronic properties, e.g., high ionization energy and low reactivity. ${ }^{1}$ The partial filling of states in a given shell follows Hund's rule ${ }^{2}$ resulting in atoms with unpaired electrons. The highest spin magnetic moment occurs at sub-shell filling, but such sub-shell filled atoms do not generally exhibit enhanced stability. In fact, for the transition metal series, the ionization potential progressively decreases or even shows a minimum through the subshell filling. ${ }^{1}$ Furthermore, the cohesive energy of solids of the $3 d$ transition metals presents a minimum in the middle of the series. ${ }^{3}$ Somewhat analogous to the case of atoms, the quantum confinement of electrons in small compact symmetric metallic clusters also results in electronic states grouped into shells with a different sequence $1 \mathrm{~S}^{2}, 1 \mathrm{P}^{6}, 1 \mathrm{D}^{10}, 2 \mathrm{~S}^{2}, \ldots$ (the electronic states in clusters are labeled by uppercase letters while the atomic states are labeled by lowercase letters) ${ }^{4-8}$ Like atoms, clusters with filled electronic shells lead to stable species as manifested through magic numbers ${ }^{9}$ observed at cluster sizes containing $2,8,18,20,34,40, \ldots$ valence electrons. This analogy, originally introduced through the electronic states in a "jellium sphere" where the confined electron gas is subjected to a uniform spherical positive background of the size of the cluster, extends beyond this oversimplified model. ${ }^{10,11}$ Numerous first principles electronic structure studies and gas phase experiments on metallic clusters have demonstrated the close grouping of

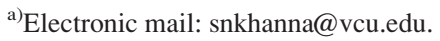

electronic states into shells and have further shown that the shapes of the cluster electronic orbitals resemble those in atoms. ${ }^{12-27}$ Moreover, experiments on the reactivity of clusters have also provided evidence that clusters with filled electronic shells exhibit reduced reactivity. For example, while bulk aluminum is readily oxidized by oxygen, an $\mathrm{Al}_{13}{ }^{-}$cluster with filled $1 \mathrm{~S}^{2}, 1 \mathrm{P}^{6}, 1 \mathrm{D}^{10}, 2 \mathrm{~S}^{2}, 1 \mathrm{~F}^{14}$, and $2 \mathrm{P}^{6}$ shells, and other species with filled shells exhibit strong resistance to etching by oxygen, typical of inert atoms. ${ }^{28}$ Motivated by these analogies, it was almost two decades ago that Khanna and Jena introduced the concept that metal clusters could be regarded as superatoms. ${ }^{29,30}$

While the electronic states in small metallic clusters are grouped into shells and the states of a given angular momentum resemble those in atoms, the filling of the electronic states in small metallic clusters generally does not follow Hund's rule of maximum multiplicity. It was pointed quite early on that clusters can undergo Jahn Teller distortions ${ }^{31-34}$ that break the degeneracy in the electronic states through geometrical distortions, and that the energy gain in the Jahn Teller distortion generally exceeds the exchange energy. Consequently, most small metallic clusters have lowest spin multiplicity with clusters of even valence count having non-magnetic ground states. One way to stabilize magnetic species is to consider compound clusters containing transition metal impurities, which can stabilize exchange splitting in two ways. One can form magnetic clusters such as $\mathrm{VCs}_{8}$, where 8 nearly free electrons fill the $1 \mathrm{~S}^{2}$ and $1 \mathrm{P}^{6}$ supershells while the remaining 5 electrons occupy the $\mathrm{V}$ centered atomic $d$-states leading to five unpaired electrons. ${ }^{35}$ An alternate approach is to induce exchange splitting in otherwise degenerate superatomic D-states through hybridization with atomic $d$-states. We recently demonstrated this intriguing possibility for the case of $\mathrm{FeMg}_{8}$, which has 26 valence electrons and forms a magnetic state with a $1 \mathrm{~S}^{2} 1 \mathrm{P}^{6}$ $1 \mathrm{D}^{10} 2 \mathrm{~S}^{2} 2 \mathrm{D}^{4}$ configuration. ${ }^{34}$ The $3 d^{10} / 1 \mathrm{D}^{10}$ and $2 \mathrm{D}^{10}$ states 
have contribution from the $d$-state of the transition metal atom and hence acquire considerable exchange splitting. It is important to underscore that this approach of inducing exchange splitting via mixing with orbitals from transition metal atoms leads to magnetic clusters that are likely to maintain exchange splitting upon assembly as the transition metal atoms occupy interior sites and are not strongly affected by cluster assembly. The purpose of this paper is to extend these earlier findings ${ }^{34}$ to a new class of superatom that has a half filled D-subshell leading to high spin multiplicity like a $\mathrm{Mn}$ atom and that also possesses energetic stability at sub-shell filling compared to its neighbors. We demonstrate this exciting finding via a $\mathrm{MnSr}_{9}$ cluster that has 5 unpaired electrons in a $2 \mathrm{D}^{5}$ shell but is still energetically stable due to $1 \mathrm{~S}^{2}, 1 \mathrm{P}^{6}, 1 \mathrm{D}^{10}$, and $2 \mathrm{~S}^{2}$ filled shells with 20 valence electrons. We also illustrate how such compound clusters offer a new class of hybridization-induced effects where the hetero atom induces exchange splitting only in selected superatomic orbitals. Here, the exchange splitting in the $\mathrm{Mn}$ atom is transmitted to exchange splitting only in S and D superatomic orbitals because of the selected hybridization with orbitals of selected parity.

\section{METHODS}

Theoretical investigations at the first principles level were carried out within the framework of generalized gradient density functional theory ${ }^{36}$ implemented in the deMon $2 \mathrm{k}$ code. ${ }^{37}$ The molecular orbitals are expressed as a linear combination of Gaussian atomic functions centered at the atomic sites. The Mn atom is described by a double- $\zeta$ valence plus polarization (DZVP) basis set optimized for generalized gradient functionals. ${ }^{38}$ The $\mathrm{Sr}$ atom is described by a 10 electron quasi-relativistic effective core potential (QECP10). ${ }^{39} \mathrm{~A}$ variational fitting of the coulomb potential was employed in order to avoid the calculation of four center electron repulsion integrals speeding up the calculations. ${ }^{40}$ To this end, the fitted density was expanded in primitive Hermite Gaussian functions by using the GEN-A2* auxiliary function set for $\mathrm{Mn}$ and Sr. For each cluster size, the ground-state configuration was obtained by starting from several initial geometries and spin multiplicities. The geometries were optimized using a Quasi-Newton Levenberg-Marquardt method in delocalized redundant coordinates without symmetry constrains. ${ }^{41}$ A frequency analysis was carried out to ascertain the stability of the ground states. For the calculation of the $\left(\mathrm{MnSr}_{9}\right)_{2}$ dimers, we used the same generalized gradient functional in the Naval Research Laboratory Molecular Orbital Library (NRLMOL) set of codes developed by Pederson and coworkers, ${ }^{42-44}$ where it is possible to investigate anti/ferromagnetic configurations. For these calculations we employed an all electron basis set of $7 s, 5 p$, and $4 d$ functions for $\mathrm{Mn}$, and $8 s, 6 p$, and $4 d$ functions for the $\mathrm{Sr}$ atom. In both cases, the basis set was supplemented with a diffuse Gaussian function to allow for additional variational freedom.

\section{RESULTS AND DISCUSSION}

The valence configuration of a $\mathrm{Sr}$ atom is $5 s^{2}$ and a $\mathrm{Sr}_{2}$ is a weakly bound molecule with a low bond strength of $0.17 \mathrm{eV} .{ }^{3}$ However, bulk strontium is a metal with a cohesive energy of $1.72 \mathrm{eV}$ per atom. ${ }^{45}$ This trend is common to other group II elements and earlier studies on $\mathrm{Be}_{\mathrm{n}}$ (Refs. 46 and 47) and $\operatorname{Mg}_{\mathrm{n}}$ (Ref. 48) clusters have shown how the metallic character in clusters rapidly builds as multiple atoms are brought together. The increase in bonding strength and the transition to metallic character is promoted via the mixing of the excited $p$-states that are unoccupied in individual atoms, with the $s$-states. For $\mathrm{Mg}_{\mathrm{n}}$ clusters, earlier studies have indicated that there is already appreciable $p$-character in the valence distribution for clusters containing as few as 5 atoms, marking the beginning of metallic character. ${ }^{48}$ The valence configuration of a $\mathrm{Mn}$ atom is $3 d^{5} 4 s^{2}$ with five unpaired electrons and a filled $d$-subshell. We wanted to examine if a compound cluster $\mathrm{MnSr}_{\mathrm{n}}$ could possess a high spin configuration as well as a closed shell of paired electrons to offer a stable magnetic superatom that has both a stable moment and energetic stability. We further investigate if such stable cluster could form assemblies maintaining their identity.

Figure 1 shows the evolution of the ground state geometry of $\mathrm{MnSr}_{\mathrm{n}}$ clusters containing 6 to $12 \mathrm{Sr}$ atoms. All the ground sates geometries of $\mathrm{MnSr}_{\mathrm{n}}$ contain the $\mathrm{Mn}$ atom enclosed in the cage of $\mathrm{Sr}$ atoms and are all compact structures with average $\mathrm{Sr}-\mathrm{Sr}$ bond length ranging from $2.91 \AA$ for the $\mathrm{MnSr}_{6}$ to $4.13 \AA$ for $\mathrm{MnSr}_{12}$ and average $\mathrm{Mn}-\mathrm{Sr}$ bond length varying between $2.87 \AA$ for $\mathrm{MnSr}_{6}$ to $3.40 \AA$ for $\mathrm{MnSr}_{12} . \mathrm{MnSr}_{6}$ presents an octahedral structure of $\mathrm{Sr}$ atoms containing an endohedral $\mathrm{Mn}$ atom. In $\mathrm{MnSr}_{7}$, the $\mathrm{Sr}$ atoms form a pentagonal $b i$-pyramid with an interior $\mathrm{Mn}$ atom. Studies where a Mn atom was forced to occupy an exterior site resulted in a structure $2.29 \mathrm{eV}$ higher in energy. The structure of $\mathrm{MnSr}_{8}$ is quite interesting. The $\mathrm{Sr}$ sites form a distorted square anti-prism with an interior $\mathrm{Mn}$ atom. The

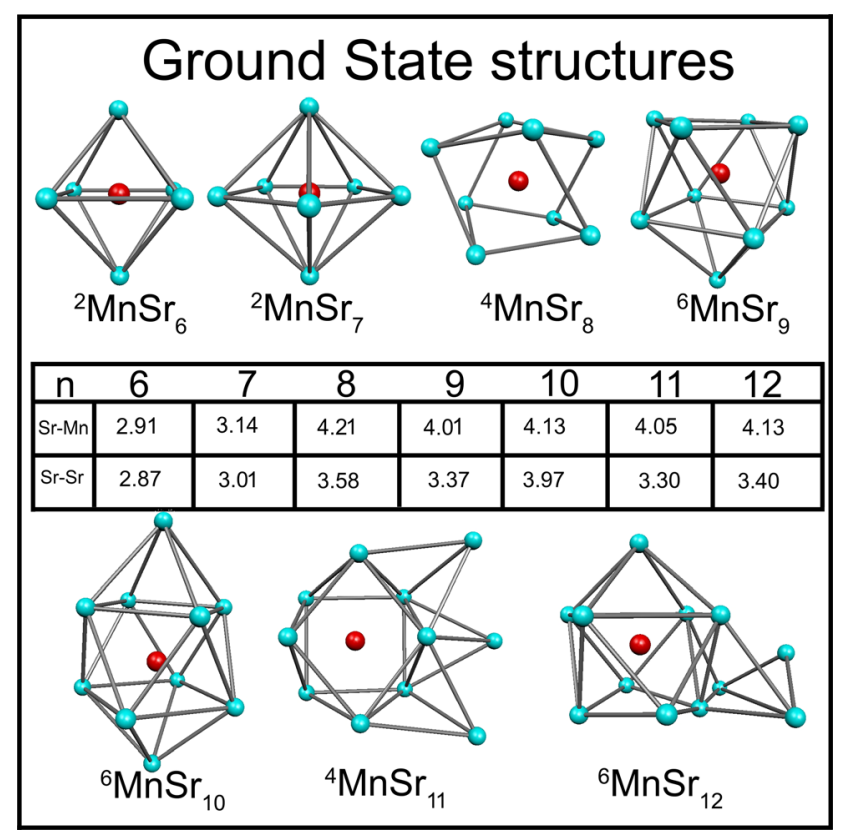

FIG. 1. The Ground state geometries of $\operatorname{MnSr}_{n}(n=6-12)$ clusters. The spin multiplicity is shown as a superscript. The cyan spheres represent the $\mathrm{Sr}$ atoms, and the red spheres represent the Mn atoms. The average Sr-Mn and Sr-Sr distances in units of $\AA$ are also given. 
cluster has a spin magnetic moment of $3 \mu_{\mathrm{b}}$. The structure of $\mathrm{MnSr}_{9}$ is a rather symmetric square anti-prism geometry of $\mathrm{Sr}$ atoms with the ninth $\mathrm{Sr}$ atom decorating one of the two parallel faces of the anti-prism. The $\mathrm{MnSr}_{10}$ structure is obtained by decorating the two opposite parallel faces of the $\mathrm{MnSr}_{8}$ anti-prism by two $\mathrm{Sr}$ atoms. $\mathrm{MnSr}_{11}$ structure does not follow the same trend shown from $\mathrm{MnSr}_{8}$ through $\mathrm{MnSr}_{10}$. But instead, it is the square anti-prism with the three remaining $\mathrm{Sr}$ atoms encrusted in the triangular adjacent faces near to each other. Finally $\mathrm{MnSr}_{12}$ follows from a $\mathrm{MnSr}_{9}$ structure plus three $\mathrm{Sr}$ atoms decorating the adjacent triangular faces of the anti-prism structure. This structure was $1.51 \mathrm{eV}$ more stable than the icosahedra of $\mathrm{Sr}$ atoms with an interior $\mathrm{Mn}$ site. It is interesting to note that $\mathrm{MnSr}_{\mathrm{n}}(\mathrm{n}=10$ 12) clusters are built on a $\mathrm{MnSr}_{9}$ core suggesting somewhat that this cluster may serve as a core for the larger sizes.

Since clusters of group II elements have weakly bound molecules and the bonding evolves towards metallic character with increasing size, we wanted to probe if $\mathrm{MnSr}_{n}$ clusters would exhibit any electronic shell effects. To this end we probed two quantities, namely, the progression of the binding energy (BE), and secondly the nature of electronic states including appearance and filling of electronic shells. We start with the progression of electronic binding as successive $\mathrm{Sr}$ atoms are added to a $\mathrm{MnSr}_{n}$ cluster. The change in binding energy was monitored through the increase in binding of the $\operatorname{MnSr}_{n}(n=6-12)$ clusters as obtained from the equation

$$
\mathrm{BE}_{\mathrm{n}}=\mathrm{E}(\mathrm{Sr})+\mathrm{E}\left(\mathrm{MnSr}_{\mathrm{n}-1}\right)-\mathrm{E}\left(\mathrm{MnSr}_{\mathrm{n}}\right)
$$

Here $\mathrm{E}(\mathrm{Sr}), \mathrm{E}\left(\mathrm{MnSr}_{\mathrm{n}-1}\right), \mathrm{E}\left(\mathrm{MnSr}_{\mathrm{n}}\right)$ are the total energies of a $\mathrm{Sr}$ atom, $\mathrm{MnSr}_{\mathrm{n}-1}$ cluster, and $\mathrm{MnSr}_{n}$ cluster, respectively. Note that $\mathrm{BE}_{n}$ represents the gain in energy as a $\mathrm{Sr}$ atom is added to the preceding size $\mathrm{MnSr}_{\mathrm{n}-1}$. Figure 2(a) shows $B_{n}$ as a function of $n$. Note that there is a distinct maximum at $\mathrm{MnSr}_{9}$. More importantly, there is a large gain in energy in going from $\mathrm{MnSr}_{7}$ to $\mathrm{MnSr}_{9}$ passing through $\mathrm{MnSr}_{8}$ and a drop thereafter. Such local maximum is indicative of especially stable species. This stability is also reflected in the electronic spectrum as seen through the progression of the HOMO-LUMO gap. A large gap is a signature of chemical stability as the system resists both accepting or donating electronic charge. Figure 2(b) shows the variation of HOMO-LUMO gap in $\mathrm{MnSr}_{n}$ clusters and $\mathrm{MnSr}_{9}$ with around $0.35 \mathrm{eV}$ is the 2nd largest HOMOLUMO gap of the series. While both $\mathrm{BE}_{\mathrm{n}}$ and HOMOLUMO gap indicate local energetic stability, there are two puzzling questions. First, a $\mathrm{MnSr}_{9}$ has 25 valence electrons (18 coming from $\mathrm{Sr}$ atoms and 7 from $\mathrm{Mn}$ atom) that do not correspond to a magic number in the conventional nearly free electron model. Second, the theoretical studies indicate that there are 5 unpaired electrons in the ground state. How does the sub-shell filling lead to an energetic stable species? The magic character of $\mathrm{MnSr}_{9}$, therefore, cannot be reconciled within the conventional nearly free electron model.

A single $\mathrm{Mn}$ atom has a spin multiplicity of 6 with 5 unpaired $d$ electrons and a $3 d^{5} 4 s^{2}$ electronic configuration.

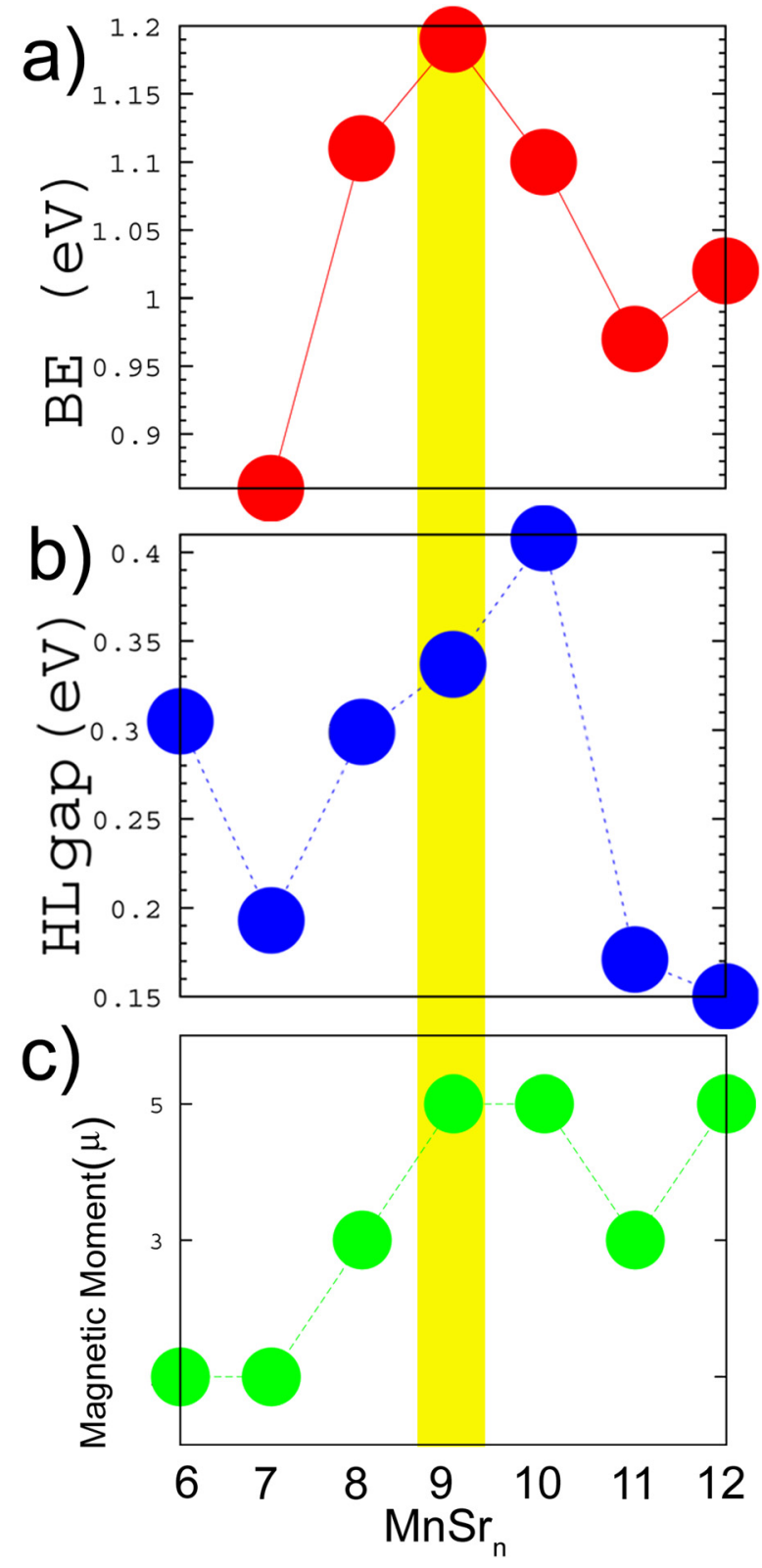

FIG. 2. Evolution of three electronic indices as a function of the number of $\mathrm{Sr}$ atoms in the $\mathrm{MnSr}_{\mathrm{n}}$ clusters. (a) the Binding Energy (BE), (b) the HOMO-LUMO gap (HL), and (c) the Magnetic Moment $\left(\mu_{\mathrm{b}}\right)$. Energies are given in units of $\mathrm{eV}$.

Our theoretical studies also indicate that the atom is marked by a large exchange splitting with unoccupied $d$-states almost $4.4 \mathrm{eV}$ higher than the majority occupied $d$-states. We therefore monitored the variation of the spin magnetic moment as successive $\mathrm{Sr}$ atoms were added to $\mathrm{MnSr}_{6}$. Figure 2(c) shows the variation of the spin magnetic moment. As a $\mathrm{Sr}$ atom is divalent while $\mathrm{Mn}$ has an odd number of electrons, the lowest magnetic moment of the cluster can only be $1.0 \mu_{\mathrm{B}}$. The addition of $\mathrm{Sr}$ atoms initially quenches the magnetic moment of the bare $\mathrm{Mn}$ atom as both $\mathrm{MnSr}_{6}$ and $\mathrm{MnSr}_{7}$ have only 1 unpaired electron. Successive addition of $\mathrm{Sr}$ then increases the number of unpaired electrons to 3 in $\mathrm{MnSr}_{8}$ while both $\mathrm{MnSr}_{9}$ and $\mathrm{MnSr}_{10}$ have a spin magnetic moment of $5.0 \mu_{\mathrm{B}}$. This increase is analogous to the filling of the $d$-shell across the $3 d$-series and is a signature of Hund's rule 
in atoms. However, what leads to this Hund's coupling in cluster electronic orbitals?

To answer this question, we examine the nature of the electronic orbitals in various clusters. Figures 3(a)-3(d) show the one-electron energy levels and the electronic orbitals associated with each level for the spin up and down configurations for $\mathrm{MnSr}_{7}, \mathrm{MnSr}_{8}, \mathrm{MnSr}_{9}$, and $\mathrm{MnSr}_{10}$, respectively. Note that $\mathrm{Mn}$ occupies a central site and its atomic $d$-orbitals hybridize to form cluster S- or D-orbitals because of symmetry. The pictures show the plots of the electron wave function that can help to identify the global nature of the orbitals. Starting with Fig. 3(a), $\mathrm{MnSr}_{7}$ has 21 valence electrons that are distributed as follows. The lowest state is spread out over several atoms and has 1S superorbital character. The first two electrons occupy majority and minority S-states. The next majority state has a $d$-character and can be classified as 1D orbital. It is primarily composed of the majority atomic $d$-states at the Mn site. The next set of majority orbitals have $1 \mathrm{P}$ and $2 \mathrm{~S}$ character accommodating 4 more electrons, and one more electron in the HOMO with $2 \mathrm{D}$ character. The minority states have $1 \mathrm{P}, 2 \mathrm{~S}$, and $1 \mathrm{D}$ character accommodating 9 electrons. The minority 1D-orbitals that accommodate 5 electrons leads to a closed $1 \mathrm{~S}^{2}, 1 \mathrm{P}^{6}$, $1 \mathrm{D}^{10}$, and $2 \mathrm{~S}^{2}$ shell with 20 valence electrons. The remaining electron occupies the next set of cluster orbitals, which are majority 2D states. It was found that $1.51 \mathrm{eV}$ separates the minority 1D-states from the majority 1D states. This large splitting is derived from the large exchange splitting of $4.4 \mathrm{eV}$ in atomic Mn. Most importantly, this splitting is also translated to 2D states where the majority spin states constitute the next set of states. Adding one $\mathrm{Sr}$ atom to $\mathrm{MnSr}_{7}$ contributes two additional valence electrons that occupy $2 \mathrm{D}$ majority states as shown in Fig. 3(b), increasing the moment to $3.0 \mu_{\mathrm{B}}$ in $\mathrm{MnSr}_{8}$. Addition of another Sr, fills the 2D subshell and results in the maximum moment of $5.0 \mu_{\mathrm{B}}$ in $\mathrm{MnSr}_{9}$ as shown in Fig. 3(c). What is interesting is that the increase

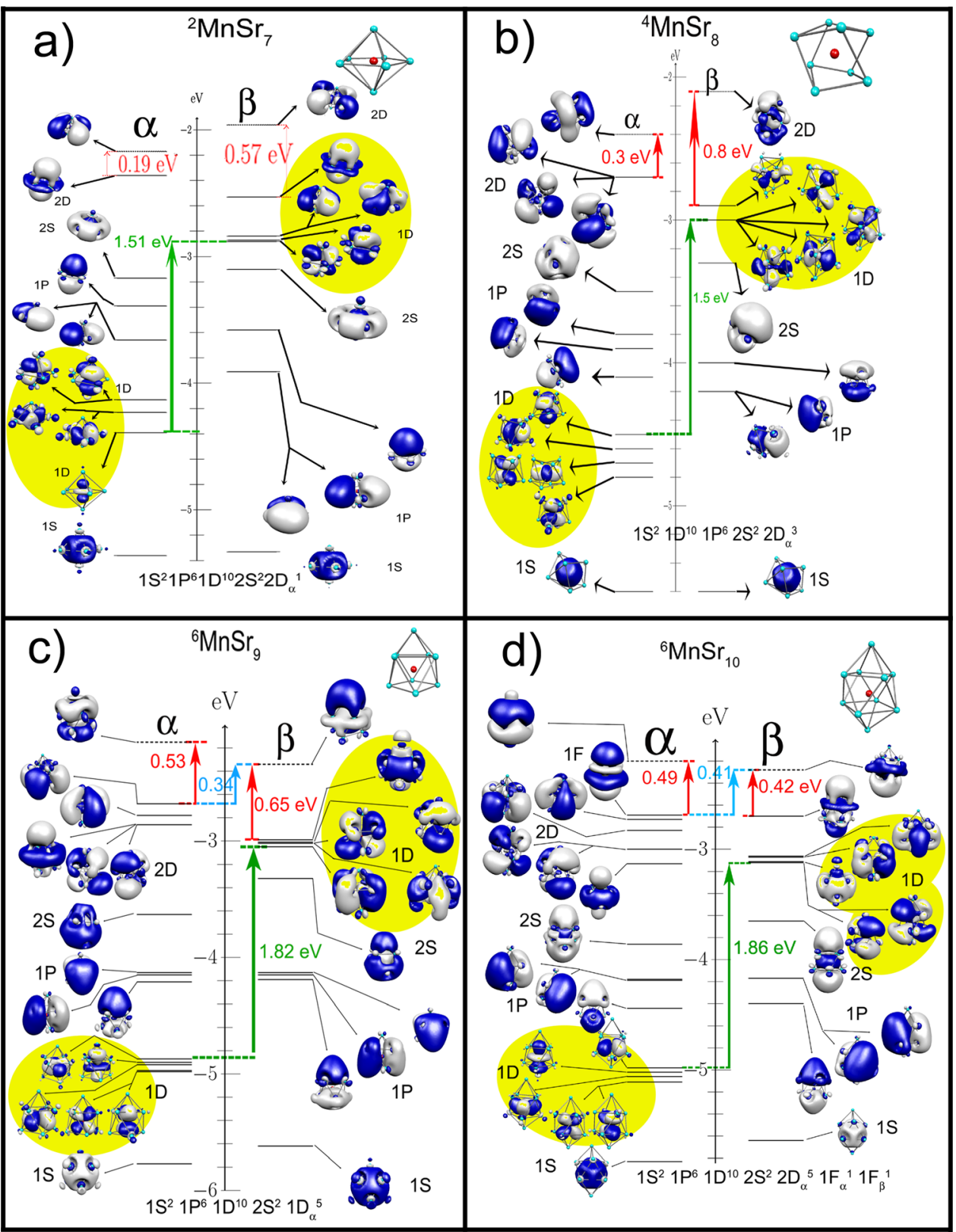

FIG. 3. One-electron energy levels and orbital wave-function isosurfaces (iso$\mathrm{val}=0.01$ a.u. $)$ of $\mathrm{Sr}_{\mathrm{n}} \mathrm{Mn}(\mathrm{n}=7-10)$ clusters. The majority $(\alpha)$ and minority $(\beta)$ levels are shown at the left and right of the energy scale arrow respectively for each cluster. The horizontal lines (continuous and dotted) represent the filled and unfilled levels respectively. For each level the angular momentum is marked. Green arrows indicate the exchange splitting of the D-orbitals, and the HOMO-LUMO is given in red and blue text. 
in moment is accompanied by the increase in binding as discussed in Fig. 2(a). $\mathrm{MnSr}_{9}$ then appears as a locally stable cluster that is also marked by high spin multiplicity and can be characterized as a magnetic superatom that can be described as a mimic of a $\mathrm{Mn}$ atom in the atomic sequence. ${ }^{25}$ Further addition of a $\mathrm{Sr}$ atom leads to filling of a $1 \mathrm{~F}$ set of states in $\mathrm{MnSr}_{10}$ as shown in Fig. 3(d). A pair of electrons occupies the $\mathrm{F}$ state and the magnetic moment remains pinned at $5.0 \mu_{\mathrm{B}}$. This brings out an interesting aspect of these mixed clusters as seen in electronic states shown in Fig. 3(c). P- or the F-states have a different symmetry and do not hybridize with the $\mathrm{Mn} d$-states. Consequently, the large exchange splitting of atomic $d$-states in Mn is only transmitted to superatomic S- and D- states. This shows the novel aspect of these compound clusters, namely, that by a proper choice of the heteroatom, one can induce splitting only in selected sets of states. In addition, by controlling the size, one can choose the character of the orbitals forming the HOMO. This subtle control of the splitting in molecular orbitals can be important in catalysis or in transport properties of such species.

In the above we have demonstrated that $\mathrm{MnSr}_{9}$ is a highly stable magnetic cluster. It is then interesting to investigate if such motifs would maintain their magnetic characteristics when assembled to form molecules, bigger clusters, and eventually large assemblies, and how do the magnetic moments couple in such aggregates? There are several approaches to make cluster assemblies from magnetic superatoms. One could isolate clusters by passivating them with organic ligands, ${ }^{49,50}$ insert them into zeolite or other cages, ${ }^{51}$ or deposit them on non-interacting substrates. ${ }^{52}$ Forming assemblies through direct deposition on substrates, however, requires the magnetic features to be maintained through the direct assembly. Consequently, we examined the evolution of the geometry and the magnetic properties as two $\mathrm{MnSr}_{9}$ clusters were brought together starting at a large separation. Figure 4 shows the two lowest energy $\left(\mathrm{MnSr}_{9}\right)_{2}$ structures and their energy dependence on the spin state. The lowest energy structure (red square on

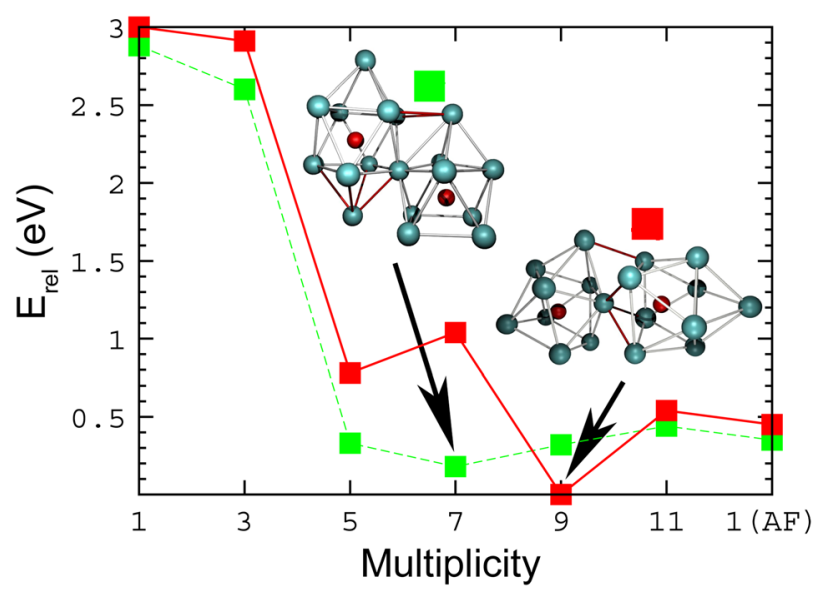

FIG. 4. Variations of the relative energies for the two lowest energy $\left(\mathrm{MnSr}_{9}\right)_{2}$ dimers as a function of their spin state. The geometry marked by a red square corresponds to the lowest energy isomer with a magnetic moment of $8 \mu_{\mathrm{B}}$, and green square corresponds to the nearest energetic structural isomer with a magnetic moment of $6 \mu_{\mathrm{B}}$. the top) is a ferromagnetic state with a magnetic moment of $8 \mu_{\mathrm{B}}$, in which the individual clusters did maintain their geometrical identity. For such an arrangement, the antiferromagnetic configuration lies $0.45 \mathrm{eV}$ above the ground state. We also found another geometrical isomer (green square on the top), that is just $0.18 \mathrm{eV}$ higher than the lowest energy structure, and has a spin moment of $6 \mu_{\mathrm{B}}$. In this isomer the $\mathrm{MnSr}_{9}$ units form a symmetric configuration sharing one $\mathrm{Sr}$ atom. These results indicate that the new $\mathrm{MnSr}_{9}$ superatoms are likely to maintain their identity upon assembly.

\section{CONCLUSION}

In summary, the present work offers a strategy to design magnetic motifs in the superatom family via doping the nonmagnetic group II atoms with a $\mathrm{Mn}$ atom. The $\mathrm{Mn}$ atom occupies the central site and the atomic Mn $d$-states hybridize with Sr states to form superatomic D-states. This hybridization results in a large exchange splitting of the D-super orbitals. For $\mathrm{MnSr}_{9}$ such a combination acquires energetic stability as well as high spin. Studies, where the two units are brought together to form a $\left(\mathrm{MnSr}_{9}\right)_{2}$ super-molecule, show that the individual clusters do retain their shapes and that the molecule is marked by a ferromagnetic ground state with an antiferromagnetic state $0.45 \mathrm{eV}$ higher in energy. While the transport through such a supermolecule will form the basis of our future work, we did examine the density of states in the ferromagnetic and antiferromagnetic configurations. These are shown in Figure 5 in which the energy levels were broadened by Gaussians of $0.1 \mathrm{eV}$ widths. As seen in Figure 5(a), the ferromagnetic state has a high spin polarization. Examination of the atomic composition of the density of states brought out an interesting feature. Figure 6 shows

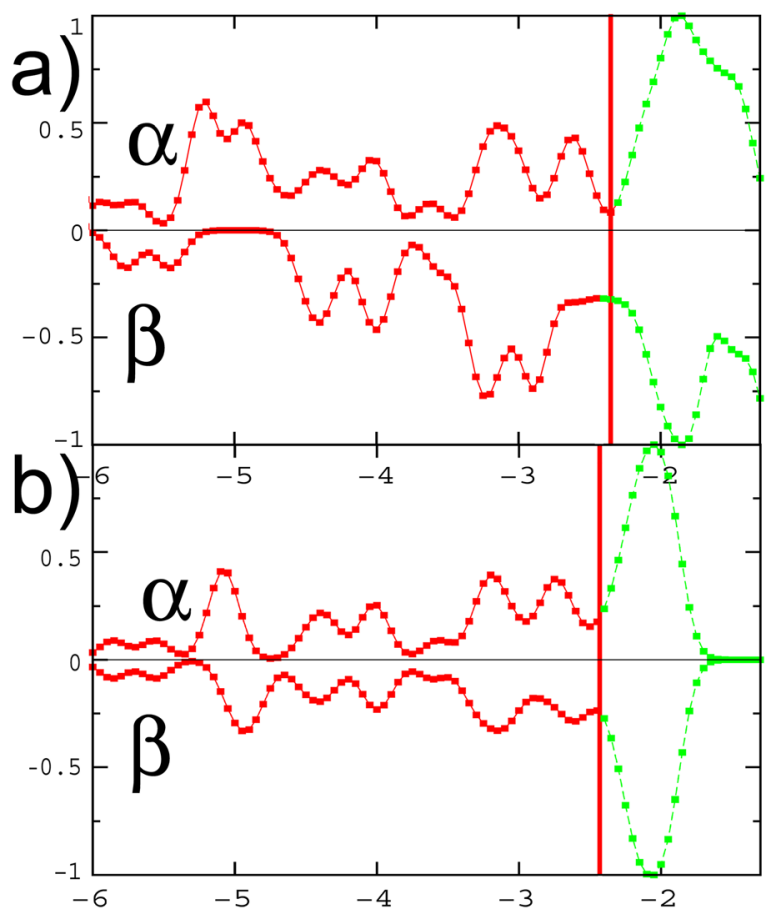

FIG. 5. Density of states for the lowest energy $\left(\mathrm{MnSr}_{9}\right)_{2}$ structure in its (a) ferromagnetic $\left(8 \mu_{\mathrm{B}}\right)$, and (b) antiferromagnetic $\left(0 \mu_{\mathrm{B}}\right)$ states. The Fermi level is marked by a vertical line. 


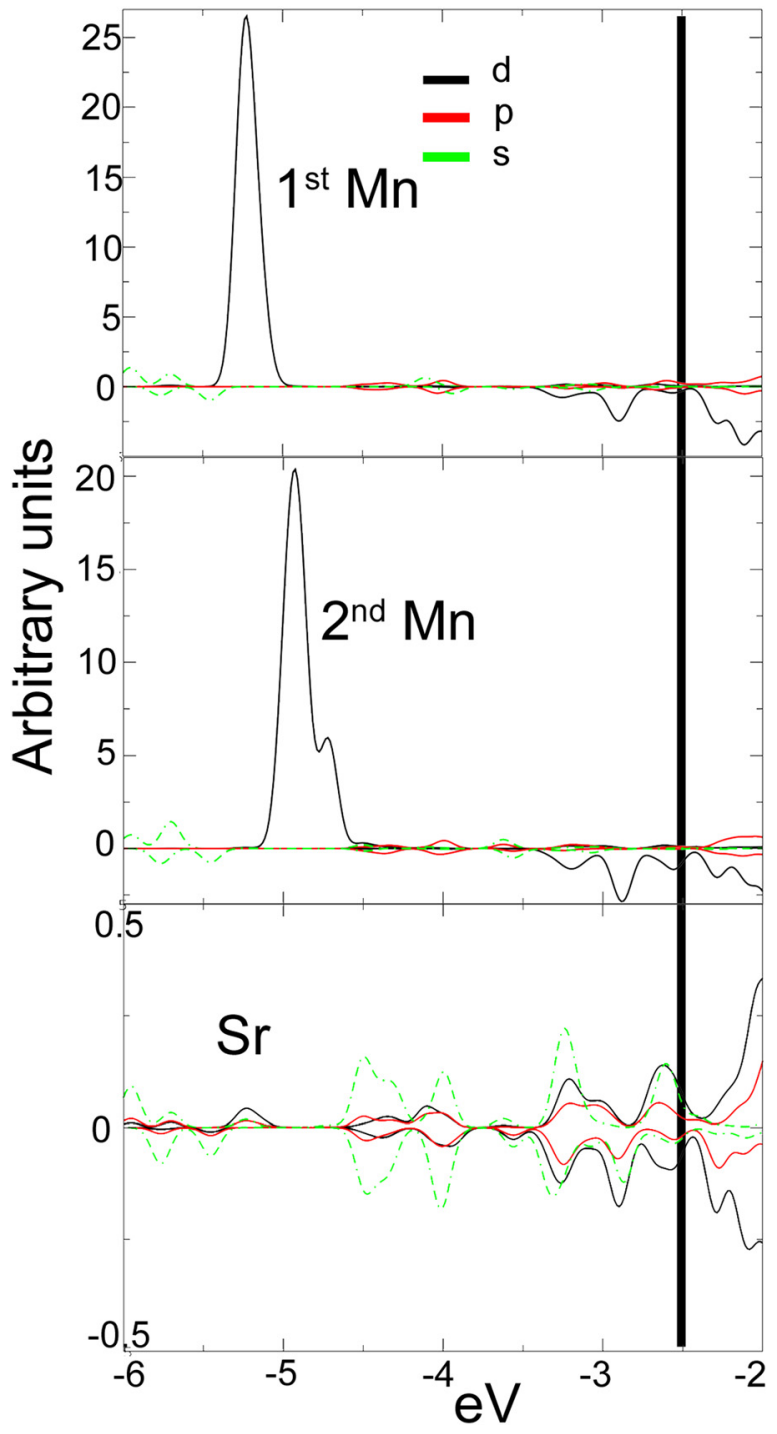

FIG. 6. Atomic projected density of states for the lowest energy ferromagnetic $\left(\mathrm{MnSr}_{9}\right)_{2}$ structure $\left(8 \mu_{\mathrm{B}}\right)$. The Fermi level is marked by a vertical line. The solid line represents the atomic $d$ contribution, and the red and green lines represent respectively the atomic $p$ and $s$ atomic contribution.

that the projected density of states at the various sites for the lowest energy ferromagnetic state. Note that the states at the Fermi level (shown by vertical line) are mostly comprised of $\mathrm{Sr}$ states (note that the density of states for $\mathrm{Sr}$ needs to be multiplied by 18 to get contributions from all $\mathrm{Sr}$ atoms). This would suggest that in any transport by such a motif, the current will be largely carried by the metallic skin of $\mathrm{Sr}$ atoms in $\left(\mathrm{MnSr}_{9}\right)_{2}$. The lack of spin orbit coupling may then allow spins to exhibit coherence over longer distances. We hope the present work will motivate the experimental work on some of the systems investigated in this work.

\section{ACKNOWLEDGMENTS}

We gratefully acknowledge support from U. S. Department of Energy (DOE) through grant DE-FG02-11ER16213.

${ }^{1}$ E. H. James, Inorganic Chemistry, 4th ed. (Harper Collins, 1993), pp. 26-38. ${ }^{2}$ N. L. Ira, Quantum Chemistry, 6th ed. (Pearson Prentice Hall, 2009), p. 332.
${ }^{3}$ CRC Handbook of Chemistry and Physics: A Ready-Reference Book of Chemical and Physics, 88th ed. (CRC, Boca Raton, FL, 2008), pp. $9-60$.

${ }^{4}$ C. Ashman, S. N. Khanna, and M. R. Pederson, Chem. Phys. Lett. 324, 137 (2000).

${ }^{5}$ B. D. Leskiw, A. W. Castleman, Jr., C. Ashman, and S. N. Khanna, J. Chem. Phys. 114, 1165 (2001).

${ }^{6}$ J. U. Reveles and S. N. Khanna, Phys. Rev. B 72, 165413 (2005).

${ }^{7}$ J. U. Reveles, S. N. Khanna, P. J. Roach, and A. W. Castleman, Jr., Proc. Natl. Acad. Sci. 103, 18405 (2006).

${ }^{8}$ A. W. Castleman, Jr. and S. N. Khanna, J. Phys. Chem. C 113, 2664 (2009). ${ }^{9}$ V. M. Medel, A. C. Reber, J. U. Reveles, and S. N. Khanna, J. Chem. Phys. 136, 134311 (2012).

${ }^{10}$ J. Hartig, A. Stösser, P. Hauser, and H. Schnöckel, Angew Chem. Int. Ed. 46, 1658 (2007).

${ }^{11}$ P. J. Roach, W. H. Woodward, A. C. Reber, S. N. Khanna, and A. W. Castleman, Jr., Phys. Rev. B 81, 195404 (2010).

${ }^{12}$ W. D. Knight, K. Clemenger, W. A. de Heer, W. A. Saunders, M. Y. Chou, and M. L. Cohen, Phys. Rev. Lett. 52, 2141 (1984).

${ }^{13}$ R. E. Leuchtner, A. C. Harms, and A. W. Castleman, Jr., J. Chem. Phys. 91, 2753 (1989).

${ }^{14}$ M. Brack, Rev. Mod. Phys. 65, 677 (1993).

${ }^{15}$ W. A. de Heer, Rev. Mod. Phys. 65, 611 (1993).

${ }^{16}$ C. Ashman, S. N. Khanna, J. Kortus, and M. R. Pederson, in Cluster and Nanostructure Interfaces, edited by P. Jena, S. N. Khanna, and B. K. Rao (World Scientific, London, 2000), pp. 383-392.

${ }^{17}$ D. E. Bergeron, A. W. Castleman, Jr., T. Morisato, and S. N. Khanna, Science 304, 84 (2004).

${ }^{18}$ D. E. Bergeron, P. J. Roach, A. W. Castleman, Jr., N. O. Jones, and S. N. Khanna, Science 307, 231 (2005).

${ }^{19}$ N. O. Jones, J. U. Reveles, S. N. Khanna, D. E. Bergeron, P. J. Roach, and A. W. Castleman, Jr., J. Chem. Phys. 124, 154311 (2006).

${ }^{20}$ A. W. Castleman, Jr., S. N. Khanna, A. Sen, A. C. Reber, M. Qian, K. M. Davis, S. J. Peppernick, A. Ugrinov, and M. D. Merritt, Nano Lett. 7, 2734 (2007).

${ }^{21}$ P. D. Jadzinsky, G. Calero, C. J. Ackerson, D. A. Bushnell, and R. D. Kornberg, Science 318, 430 (2007).

${ }^{22}$ M. Walter, J. Akola, O. Lopez-Acevedo, P. D. Jadzinsky, G. Calero, C. J. Ackerson, R. L. Whetten, H. Gronbeck, and H. Hakkinen, Proc. Natl. Acad. Sci. U.S.A. 105, 9157 (2008).

${ }^{23}$ K. Pradhan, P. Sen, J. U. Reveles, and S. N. Khanna, J. Phys.: Condens. Matter 20, 255243 (2008).

${ }^{24}$ J. U. Reveles, P. A. Clayborne, A. C. Reber, S. N. Khanna, K. Pradhan, P. Sen, and M. R. Pederson. Nat. Chem. 1, 310 (2009).

${ }^{25}$ K. Pradhan, J. U. Reveles, P. Sen, and S. N. Khanna, J. Chem. Phys. 132, 124302 (2010).

${ }^{26}$ A. C. Reber, S. N. Khanna, P. J. Roach, W. H. Woodward, and A. W. Castleman Jr., J. Phys. Chem. A 114, 6071 (2010).

${ }^{27}$ U. Gupta, J. U. Reveles, J. J. Melko, S. N. Khanna, and A. W. Castleman, Jr., J. Phys Chem. C 114, 15963 (2010).

${ }^{28}$ R. Burgert, H. Schnoeckel, A. Grubisic, X. Li, S. T. Stokes, K. H. Bowen, G. F. Gantefor, B. Kiran, and P. Jena, Science 319, 438 (2008).

${ }^{29}$ S. N. Khanna and P. Jena, Phys, Rev. Lett. 69, 1664 (1992).

${ }^{30}$ S. N. Khanna and P. Jena, Phys. Rev. B 51, 13705 (1995).

${ }^{31}$ H. A. Jahn and E. Teller, Proc. R. Soc. London, Ser. A 161, 220 (1937).

${ }^{32}$ K. Clemenger, Phys. Rev. B 32, 1359 (1985).

${ }^{33}$ S. N. Khanna, B. K. Rao, P. Jena, and J. L. Martins, in Physics and Chemistry of Small Clusters, edited by P. Jena, B. K. Rao, and S. N. Khanna (Plenum, New York, 1986), pp. 435-438.

${ }^{34}$ V. Medel, J. U. Reveles, S. N. Khanna, V. Chauhan, P. Sen, and A. W. Castleman, Jr., Proc. Natl. Acad. Sci. 108, 10062 (2011).

${ }^{35}$ H. He, R. Pandey, J. U. Reveles, S. N. Khanna, and S. P. Karna, Appl. Phys. Lett. 95, 192104 (2009).

${ }^{36}$ J. P. Perdew, K. Burke, and M. Ernzerhof, Phys. Rev. Lett. 77, 3865 (1996).

${ }^{37}$ A. M. Köster, P. Calaminici, M. E. Casida, V. D. Dominguez, R. FloresMoreno, G. U. Gamboa, A. Goursot, T. Heine, A. Ipatov, F. Janetzko, J. M. del Campo, J. U. Reveles, A. Vela, B. Zuniga-Gutierrez, and D. R. Salahub, deMon2k, version 2, The deMon developers Cinvestav, Mexico City (2006).

${ }^{38}$ P. Calaminici, F. Janetzko, A. M. Köster, R. Mejia-Olvera, and B. ZuñigaGutierrez, J. Chem. Phys. 126, 044108 (2007).

${ }^{39}$ D. Andrae, U. Haeussermann, M. Dolg, H. Stoll, and H. Preuss, Theory Chim. Acta 77, 123 (1990). 
${ }^{40}$ B. I. Dunlap, J. W. D. Connolly, and J. R. Sabin, J. Chem. Phys. 71, 3396 (1979).

${ }^{41}$ J. U. Reveles and A. M. Koster, J. Comput. Chem. 25, 1109 (2004).

${ }^{42}$ M. R. Pederson and K. A. Jackson, Phys. Rev. B 41, 7453 (1990).

${ }^{43}$ K. Jackson and M. R. Pederson, Phys. Rev. B 42, 3276 (1990).

${ }^{44}$ D. Porezag and M. R. Pederson, Phys. Rev. A 60, 2840 (1999).

${ }^{45}$ J. A. Moriarty, Phys. Rev. B 19, 609 (1979).

${ }^{46}$ S. N. Khanna, F. Reuse, and J. Buttet, Phys. Rev. Lett. 61, 535 (1988).

${ }^{47}$ J. M. Merritt, V. E. Bondeybey, and M. C. Heaven, Science 324, 1548 (2009).
${ }^{48}$ J. Jellinek and P. H. Acioli, J. Phys. Chem. A 106, 10919 (2002).

${ }^{49}$ M. Zhu, C. M. Aikens, M. P. Hendrich, R. Gupta, H. Qian, G. C. Schatz, and R. Jin, J. Am. Chem. Soc. 131, 2490 (2009).

${ }^{50}$ H. Hakkinen, Nat. Chem. 4, 443 (2012).

${ }^{51}$ M. N. Mikhailov, L. M. Kustov, and V. B. Kazansky, Catal. Lett. 120, 8 (2008).

${ }^{52}$ A. Perez, P. Melinon, V. Dupuis, P. Jensen, B. Prevel, J. Tuaillon, L. Bardotti, C. Martet, M. Treilleux, M. Broyer, M. Pellarin, J. L. Vaille, B. Palpant, and J. Lerme, J. Phys. D: Appl. Phys. 30, 709 (1997). 